\title{
Successful personalized chemotherapy for metastatic gastric cancer based on quantitative BRCA1 mRNA expression level: A case report
}

\author{
YING HUANG, PUYUAN WU, BAORUI LIU and JUAN DU \\ The Comprehensive Cancer Center of Drum Tower Hospital, \\ Medical School of Nanjing University and Clinical Cancer Institute of Nanjing University, \\ Nanjing, Jiangsu 210008, P.R. China
}

Received March 17, 2015; Accepted April 1, 2016

DOI: $10.3892 / 01.2016 .4546$

\begin{abstract}
Personalized chemotherapy is based on the specific genetic profile of individual patients and is replacing the traditional 'one size fits all' medicine. Breast cancer 1 (BRCA1) plays a central role in the chemotherapy-induced DNA damage response. It has been repeatedly demonstrated that BRCA1 mRNA levels were negatively associated with cisplatin sensitivity, but positively associated with docetaxel sensitivity in patients with gastric cancer in experimental and clinical studies. This feature leads to customized chemotherapy based on the BRCA1 mRNA expression level and results in a high efficacy of treatment. The present study describes the case of a 77-year-old patient with metastatic gastric cancer who was treated with personalized chemotherapy based on quantitative BRCA1 mRNA expression level. This study and the available literature data suggest that the expression level of BRCA1 mRNA is dynamic to BRCA1-based chemotherapy. More importantly, de novo assessment of BRCA1 status is a preferable option for ciscisplatin- or docetaxel-resistant patients, since the expression levels of BRCA1 mRNA in certain patients may alter significantly following treatment. Therefore, BRCA1 expression should be assessed for predicting differential chemosensitivity and tailoring chemotherapy in gastric cancer.
\end{abstract}

\section{Introduction}

The median overall survival (OS) time of patients with metastatic gastric cancer is only $\sim 4$ months, and the median overall survival time of such patients who are $>75$ years old

Correspondence to: Dr Juan Du, The Comprehensive Cancer Center of Drum Tower Hospital, Medical School of Nanjing University and Clinical Cancer Institute of Nanjing University, 321 Zhongshan Road, Nanjing, Jiangsu 210008, P.R. China

E-mail: dujuanglyy@hotmail.com

Key words: BRCA1, real-time gene expression, personalized chemotherapy, metastatic gastric cancer is $<3$ months (1). Personalized chemotherapy delivers the appropriate care to the correct patient at the right time and results in measurable improvements in outcomes (2). Breast cancer 1 (BRCA1) acts mainly in the response to DNA damage by participating in DNA repair, cell cycle regulation, mRNA transcription, and protein ubiquitination and SUMOylation, and in the response to antimicrotubules by regulating the spindle checkpoint (3). A growing amount of evidence has indicated that low BRCA1 expression confers increased sensitivity to DNA-damaging chemotherapeutic agents, including cisplatin and oxaliplatin, while high BRCA1 expression leads to sensitivity to mitotic spindle poisons, such as docetaxel (4). The BRCA1 gene has been identified as a useful predictive marker for chemotherapeutic choice in breast cancer, ovarian cancer or non-small cell lung cancer (NSCLC). A recent study showed a beneficial effect of second-line docetaxel-based chemotherapy on the survival of patients with advanced-stage gastric cancer (5). Another study indicated that plasma BRCA1 mRNA expression levels may also have a promising role as potential predictive biomarkers for chemotherapy (6). The present study describes BRCA1-specific gastric cancer in a patient who was twice treated with personalized chemotherapy base on quantitative gene expression data. Written informed consent was obtained from the patient for publication of this case report and any accompanying images.

\section{Case report}

A 77-year-old man presented to Nanjing Drum Tower Hospital (Nanjing, Jiangsu, China) on May 21, 2013, due to epigastric discomfort, bloating and melena that had persisted for 1 month. Gastroscopy demonstrated a huge ulcer in the gastric cardia and body. Examination of a biopsy specimen showed the presence of poorly differentiated adenocarcinoma. The expression of human epidermal growth factor receptor-2 was negative.Computed tomography scans revealed massive liver metastases (Fig. 1A). The largest metastatic lesions were located in the right lobe of the liver and its diameter was $\sim 1.3 \mathrm{~cm}$. The patient's carbohydrate antigen (CA) 19-9 tumor marker level was 9,736 U/ml (normal range, 0-39 U/ml). Other tumor markers, including CA12-5, CA242, CA72-4, 

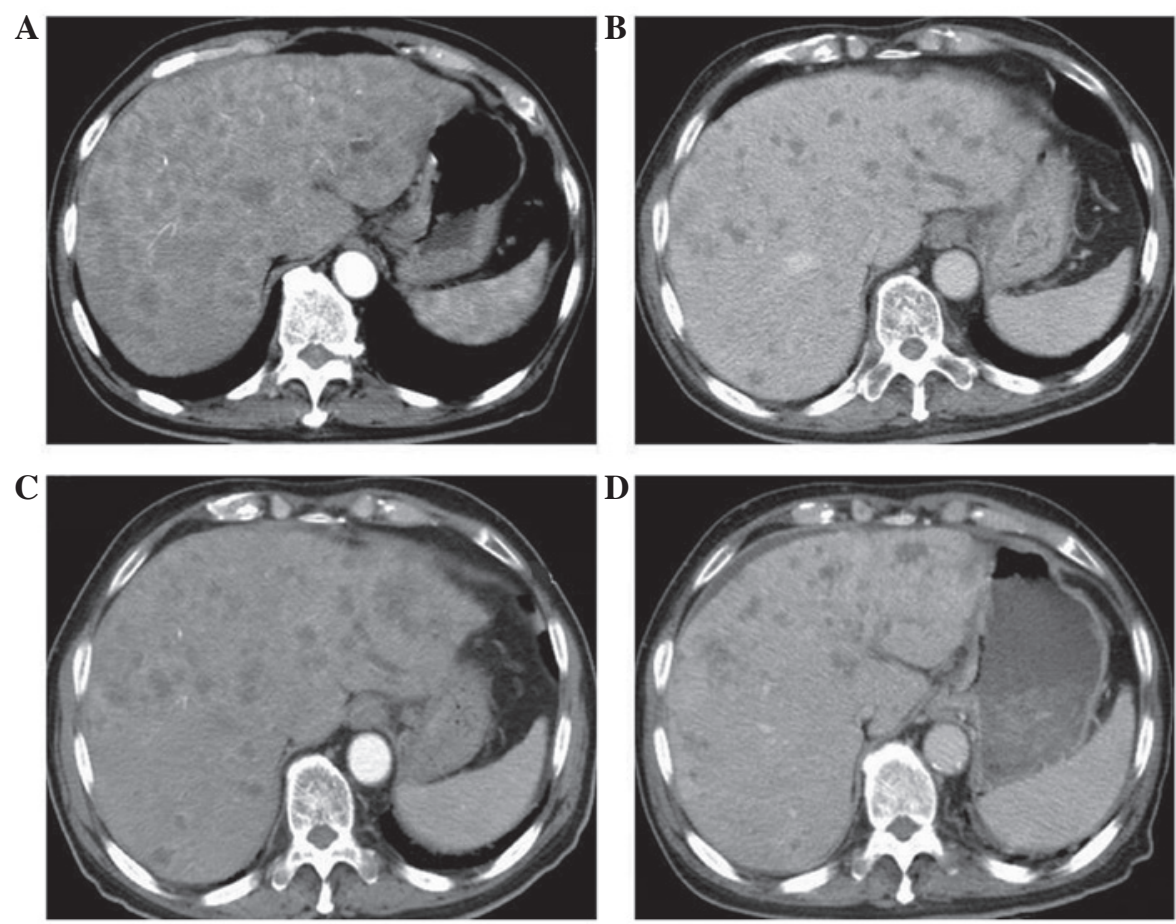

Figure 1. Abdominal and chest computed tomography scans revealing (A) multiple and massive liver metastases prior to therapy; (B) partial remission of the metastatic liver lesions after 2 cycles of docetaxel + calcium folinate + 5-fluorouracil chemotherapy; (C) progression of the metastatic liver lesions following 7 cycles of treatment; and (D) stable liver lesions following 2 cycles of oxaliplatin and S-1 chemotherapy.
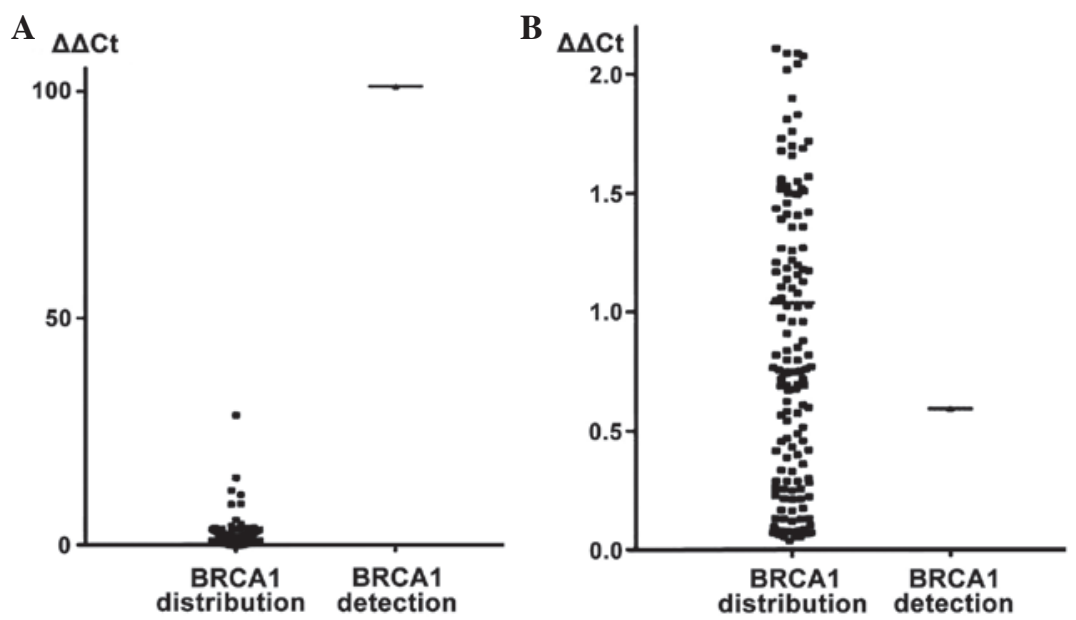

Figure 2. (A) BRCA1 mRNA expression at first time (March 2013) showing high levels (>100). (B) BRCA1 mRNA expression later (February 2014) showing low levels (0.59). BRCA1, breast cancer 1 .

carcinoembryonic antigen and alpha-fetoprotein were tested. CA12-5 (59.7 U/ml; normal range, 0-30.2 U/ml) and CA242 (27.9 U/ml; normal range, $0-15 \mathrm{U} / \mathrm{ml}$ ) levels were slightly abnormal. The medical history included a 10-year history of hypertension and type 2 diabetes, and surgeries consisting of a cholecystectomy in 1989, prostatectomy in 2010 and phacoemulsification in 2013. The current diagnosis was of advanced gastric cancer with liver metastases.

A high BRCA1 mRNA expression level in the peripheral blood was first detected in March 2013 by quantitative polymerase chain reaction (qPCR) (Fig. 2A). Briefly, RNA was extracted using a proteinase K-containing buffer (Applied Biosystems; Thermo Fisher Scientific, Inc., Waltham, MA,
USA) and treated with DNAse I (Applied Biosystems; Thermo Fisher Scientific, Inc.). Template cDNA was amplified with specific primers and probes for $\beta$-actin and BRCA1 and Taqman Universal Master Mix (Applied Biosystems; Thermo Fisher Scientific, Inc.). The primers and probes (Applied Biosystems; Thermo Fisher Scientific, Inc.) were as follows: $\beta$-actin, forward 5'-TGAGCGCGGCTACAG CTT-3', reverse 5'-TCCTTAATGTCACGCACGATTT-3' and probe 6FAM-5'-ACCACCACGGCCGAGCGG-3'-TAMRA; BRCA1, forward 5'-GGCTATCCTCTCAGAGTGACATTT TA-3', reverse 5'-GCTTTATCAGGTTATGTTGCATGG T-3' and probe 6FAM-5'-CCACTCAGCAGAGGG-3'-MGB. qPCR was performed using the ABI Prism 7900HT Sequence 


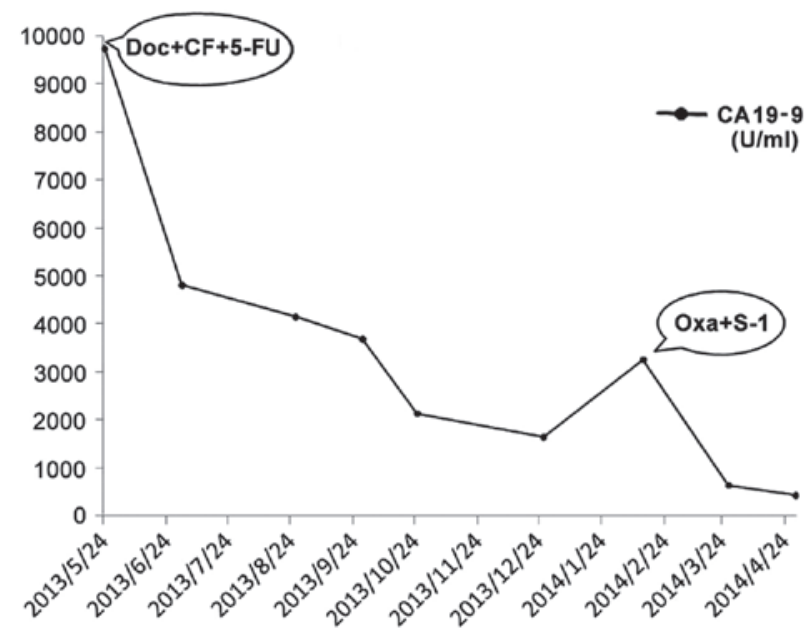

Figure 3. Analysis of CA19-9 serum level. A clear reduction in marker serum level was observed following 7 cycles of treatment with Doc $+\mathrm{CF}+5$-FU chemotherapy. This level remained within the normal range for almost 8 months. This was followed by a rebound in the levels of CA19-9, and the patient was administered a different chemotherapy regimen consisting of Oxa + S-1. The CA19-9 serum level consequently decreased once again. CA19-9, carbohydrate antigen 19-9; Doc, docetaxel; CF, calcium folinate; 5-FU, 5-fluorouracil; Oxa, oxaliplatin.

Detection System (Applied Biosystems ${ }^{\circledR}$; Thermo Fisher Scientific, Inc.) under the following conditions: $50^{\circ} \mathrm{C}$ for $2 \mathrm{~min}, 95^{\circ} \mathrm{C}$ for $10 \mathrm{~min}$, followed by 45 cycles at $95^{\circ} \mathrm{C}$ for $15 \mathrm{sec}$ and $60^{\circ} \mathrm{C}$ for $1 \mathrm{~min}$. The experiments were performed three times and the quantification of relative gene expression was according to the comparative $\mathrm{Cq}$ method using $\beta$-actin as an endogenous control (7). According to this methodology, final values were determined using the formula $2-{ }^{\Delta \Delta \mathrm{Cq}}=2-(\Delta \mathrm{Cq}$ sample $-\Delta \mathrm{Cq}$ calibrator), where $\Delta \mathrm{Cq}$ values of the calibrator and sample are determined by subtracting the $\mathrm{Cq}$ value of the target gene from the value of the $\beta$-actin gene. All analyses were performed at The Comprehensive Cancer Center of Nanjing Drum Tower Hospital, The Affiliated Hospital of Nanjing University Medical School (Nanjing, China).

Due to the high BRAC1 mRNA levels, the patient was administered personalized chemotherapy using a docetaxel + calcium folinate +5 -fluorouracil $(5$-FU) drug regimen (docetaxel, $35 \mathrm{mg} / \mathrm{m}^{2}$ on day 1 ; folinic acid, $200 \mathrm{mg} / \mathrm{m}^{2}$ as a $2 \mathrm{~h}$ infusion on day 1; 5-FU, $600 \mathrm{mg} / \mathrm{m}^{2} 22 \mathrm{~h}$ infusion on days 1 and 2; every two weeks). After 2 cycles of chemotherapy, the metastatic liver lesions responded partially (Fig. 1B) and the level of tumor marker CA19-9 concomitantly declined markedly (4146 U/ml). However, the metastatic liver lesions progressed after 7 cycles of treatment (Fig. 1C), and at the same time, there was a rebound in the level of the tumor marker (CA19-9, $3252 \mathrm{U} / \mathrm{ml})$. qPCR was performed again in February 2014 for BRCA1 mRNA level, and the result was completely different from the first time, showing that the BRCA1 mRNA expression level was now low (Fig. 2B). The patient was subsequently administered further personalized chemotherapy using a combination of oxaliplatin and S-1 (oxaliplatin, $85 \mathrm{mg} / \mathrm{m}^{2}$ on day $1 ; \mathrm{S}-1,40 \mathrm{mg} / \mathrm{m}^{2}$ twice a day on day 1-4; every two weeks). As a consequence, the metastatic liver lesions stabilized again (Fig. 1D). The levels of tumor marker during the whole treatment are shown in Fig. 3. Since April 2014 the patient was yet to receive two cycles of oxaliplatin and S-1 chemotherapy. The patient refused further treatment and was lost to follow-up.

\section{Discussion}

The present study reports a case of personalized chemotherapy based on quantitative gene expression data. The patient was $>75$ years old, had presented with several primary diseases and was in poor health. The patient has currently remained alive for almost 2 years post-diagnosis of advanced gastric cancer. The clinical benefits that were obtained from personalized chemotherapy based on BRCA1 mRNA expression is notable. Certain studies have shown that BRCA1 mRNA expression modulates chemosensitivity to chemotherapeutic agents in DNA repair, by recruiting BRCA1-A complex subunit RAP80, protein inhibitor of activated STAT family of proteins and BRCA1/BRCA1-associated RING domain 1 heterodimer (8). It is important for patients to receive secondary detection of BRAC1 mRNA expression levels in peripheral blood. qPCR tests for secondary BRCA1 mRNA expression in docetaxel-treated cancers with high BRCA1 expression may be clinically important, as tumors with altered BRCA1 expression, such as in the present study, are likely to be sensitive to oxaliplatin. Quinn et al (4) suggest that sporadic epithelial ovarian cancer patients with low levels of BRCA1 mRNA expression may obtain therapeutic benefit from platinum chemotherapy agents, whereas patients with higher levels of BRCA1 potentially have an improved response to regimens containing taxanes. Larger clinical studies are warranted to further determine the prevalence and clinical significance of the secondary expression of BRCA1 in BRCA1-specific cancer. The present findings could have implications for gastric cancers with regard to gene detection and clinical application. Moreover, altered BRCA1 expression may predict differential chemosensitivity to different anticancer drugs, which is advantageous when compared with molecular-targeted therapy. For example, a comprehensive review has shown that k-RAS mutations confer a high level of resistance to EGFR-targeting strategies in patients with either metastatic colorectal cancer or NSCLC (9). Therefore, BRCA1 may not only be associated with chemotherapeutic sensitivity to first-line treatment, but 
may also be a useful and quantitative biomarker for acquired BRCA1-resistant cancer, which should enhance efficiency in customized chemotherapy.

In the present case, the clinical benefits achieved by an older patient who was in poor bad health indicated that customized chemotherapy based on the monitoring of quantitative BRCA1 mRNA expression may provide a promising treatment for those patients presenting with metastatic gastric cancer, even following resistance.

\section{References}

1. Yang D, Hendifar A, Lenz C, Togawa K, Lenz F, Lurje G, Pohl A, Winder T, Ning Y, Groshen S and Lenz HJ: Survival of metastatic gastric cancer: Significance of age, sex and race/ethnicity. J Gastrointest Oncol 2: 77-84, 2011.

2. Ng PC, Murray SS, Levy S and Venter JC: An agenda for personalized medicine. Nature 461: 724-726, 2009.

3. Morris JR, Boutell C, Keppler M, Densham R, Weekes D, Alamshah A, Butler L, Galanty Y, Pangon L, Kiuchi T, et al: The SUMO modification pathway is involved in the BRCA1 response to genotoxic stress. Nature 462: 886-890, 2009.
4. Quinn JE, James CR, Stewart GE, Mulligan JM, White P, Chang GK, Mullan PB, Johnston PG, Wilson RH and Harkin DP: BRCA1 mRNA expression levels predict for overall survival in ovarian cancer after chemotherapy. Clin Cancer Res 13: 7413-7420, 2007.

5. Wei J, Costa C, Ding Y, Zou Z, Yu L, Sanchez JJ, Qian X, Chen H, Gimenez-Capitan A, Meng F, et al: mRNA expression of BRCA1, PIAS1 and PIAS4 and survival after second-line docetaxel in advanced gastric cancer. J Natl Cancer Inst 103: 1552-1556, 2011.

6. Shen J, Wei J, Guan W, Wang H, Ding Y, Qian X, Yu L, Zou Z, Xie L, Costa C, et al: Plasma mRNA expression levels of BRCA1 and TS as potential predictive biomarkers for chemotherapy in gastric cancer. J Transl Med 12: 355, 2014.

7. Livak KJ and Schmittgen TD: Analysis of relative gene expression data using real-time quantitative PCR and the 2(-Delta Delta C(T)) Method. Methods 25: 402-408, 2001.

8. Sobhian B, Shao G, Lilli DR, Culhane AC, Moreau LA, Xia B, Livingston DM and Greenberg RA: RAP80 targets BRCA1 to specific ubiquitin structures at DNA damage sites. Science 316: 1198-1202, 2007.

9. Linardou H, Dahabreh IJ, Kanaloupiti D, Siannis F, Bafaloukos D, Kosmidis P, Papadimitriou CA and Murray S: Assessment of somatic k-RAS mutations as a mechanism associated with resistance to EGFR-targeted agents: A systematic review and meta-analysis of studies in advanced non-small-cell lung cancer and metastatic colorectal cancer. Lancet Oncol 9: 962-972, 2008. 